\title{
NUMERICAL SOLUTIONS OF 2-D UNSTEADY INCOMPRESSIBLE FLOW IN A DRIVEN SQUARE CAVITY USING STREAMFUNCTION-VORTICITY FORMULATION
}

\author{
Vusala Ambethkar ${ }^{1 \S}$, Manoj Kumar ${ }^{2}$, Mohit Kumar Srivastava ${ }^{3}$ \\ ${ }^{1,2}$ Department of Mathematics \\ Faculty of Mathematical Sciences \\ University of Delhi \\ Delhi, 110007, INDIA \\ ${ }^{3}$ Ambedkar Institute of Advanced Communication \\ Technologies and Research \\ Delhi, 110031, INDIA
}

\begin{abstract}
In this paper, we have used a streamfunction-vorticity $(\psi-\xi)$ formulation to investigate the problem of 2-D unsteady viscous incompressible flow in a driven square cavity with moving top and bottom walls. We used this formulation to solve the governing equations along with no-slip and slip wall boundary conditions. A general algorithm was used for this formulation in order to compute the numerical solutions for the flow variables: streamfunction $\psi$, vorticityfunction $\xi$ for low Reynolds numbers $R e \leq 50$. We have executed this with the aid of a computer programme developed and run in $\mathrm{C}++$ compiler. We have proved the stability and convergence of the numerical scheme using matrix method. Following this, the stability conditions obtained for the time and space steps have been used in numerical computations to arrive at the numerical solutions with desired accuracy. Also investigated was the variation of $u$ and $v$ components of velocity at points closed to left side, top and bottom walls of the square cavity at different time levels for Reynolds number 50. Based on the numerical computations of $u$-velocity, we found: (i) the $u$-velocity is almost same near the top and bottom wall of the square cavity above and below the geometric center for $R e=15$ and 30; (ii) the absolute value
\end{abstract}

Received: September 2, 2016

(C) 2016 Academic Publications

${ }^{\S}$ Correspondence author 
of the $u$-velocity first decreases, then increases, and finally decreases, near top and bottom wall of the square cavity. Based on the numerical computations of the $v$-velocity, we found: (i) the absolute value of the $v$-velocity increases uniformly as time level increases' (ii) the $v$-velocity at the right wall of the square cavity attains a maximum value, and then, decreases towards the geometric center. The numerical solutions of the vorticity vector $\xi$ at $R e=50$ for different times along the horizontal line through geometric center of the square cavity indicate the following: (i) the vorticity contours decreased in between the left wall boundary to the midpoint of the square domain; (ii) it, then, increased in between the midpoint to the right wall boundary. Based on the numerical solutions of streamfunction $\psi$, we found: (i) the size of streamline contours decreased when the time increased; (ii) two streamline contours are generated, one above the geometric center, and the other, below the geometric center in clockwise direction.

AMS Subject Classification: 35Q30, 76D05, 76D07, 76M20

Key Words: streamfunction-vorticity formulation, unsteady incompressible flow, numerical solutions, driven square cavity, streamline contours, vorticity vector, components of velocity, low Reynolds number, no-Slip and Slip boundary conditions

\section{Introduction}

The problem of 2-D unsteady viscous incompressible flow in a square cavity plays an important role in a number of industrial contexts. For example, there is a direct relevance of cavity flows to coaters and in melt spinning processes used to manufacture micro-crystalline material. However, in our view the overwhelming importance of these flows is basic to the study of fluid mechanics. The eddy structures found in driven-cavity flows give insight into the behaviour of such structures in diverse applications. We cite the example of drag-reducing riblets and mixing cavities used to synthesize fine, polymeric composites. The streamfunction-vorticity $(\psi-\xi)$ formulation is a powerful approach for solving the two-dimensional viscous incompressible flow. It has been used in several applications such as the modeling of turbulent flows, analysis of laminar to turbulent flow transition and studies on free and mixed convection. Since the primary attractive feature of the streamfunction-vorticity method is that it does not involve the solution of the pressure field, the advantages in using this method for 2-D flow computations are manifold. However, this formulation has its share of drawbacks as well. The main drawback is that it cannot be 
applied to 3-D flow problems. Secondly, it requires the estimation of vorticity at the boundaries due to lack of boundary condition for the vorticity at no-slip boundary, for computing the flow solution in the whole domain.

Briefly discussing the related literature: Ghia et al. [1] have used the vorticity-streamfunction formulation for the two-dimensional incompressible Naiver-Stokes equations to study the effectiveness of the coupled strongly implicit multi-grid (CSI-MG) method in the determination of high-Re fine-mesh flow solutions. Schreiber and Keller [2] described a combination of numerical techniques for solving the steady plane incompressible Navier-Stokes equations and, as an illustration, used them to compute accurate solutions for the driven cavity flow problems. Fusegi and Bakhtierfarouk [4] investigated some predictions of fluid flow and heat transfer using the vorticity-velocity formulation. The problem of steady incompressible Navier-Stokes equations of a 2-D driven cavity flow with an efficient method has been investigated by Bruneau and Jouron [6]. Tezduyar et al. [7] have investigated the solution techniques using streamfunction-vorticity method of an unsteady 2-D incompressible flows. Aidun et al. [8] have investigated using flow visualization studies the global stability of a lid-driven cavity flow. Auto-Processing of very fine scale composite materials by chaotic mixing of melts has been investigated by Zumbrunnen et al. [9]. Shankar and Deshpande [11] gave an extensive of fluid mechanics in a driven cavity problem. Kalita et al. [12] have computed the flow in a thermally driven square cavity with adiabatic top and bottom walls and differentially heated vertical walls for a wide range of Rayleigh numbers. Kalita et al. [13] have proposed a higher order compact (HOC) finite difference solution procedure for the steady two-dimensional convection-diffusion equations on non uniform orthogonal cartesian grids. Kalita et al. $[12,13]$ have investigated steady state natural convection in a square cavity as well as convection-diffusion problem using fully compact higher order transformation free HOC scheme. Shokouhmand and Sayehvand [14] have studied numerically the flow and heat transfer in a square driven cavity. Erturk et al. [15] have studied the numerical solutions of 2-D steady incompressible driven cavity at high Reynolds numbers. Numerical simulations of the 2-D lid-driven cavity flow were performed for a wide range of Reynolds numbers by Bruneau and Saad [16]. Salem [17] investigated the numerical solutions of the incompressible Navier-Stokes equations in primitive variables using grid generation techniques. The $(9,5)$ HOC formulation for the transient Navier-Stokes equations in primitive variable was discussed by Kalita and Sen [18]. Discussion of transition from laminar to turbulent flow at Reynolds number starting from 1000 has been investigated by Erturk [21]. Hou [22] studied stable fourth-order streamfunction method for 
incompressible flow with boundaries. Two dimensional lid-driven cavity flow has been dealt numerically using streamfunction formulation by Poochinapan [24]. Numerical solutions of 2-D driven cavity flow with wavy bottom surface has been investigated by Uddin and Saha [25].

The literature survey pertinent to 2-D unsteady incompressible flow in a driven square cavity revealed that, to obtain highly accurate and efficient numerical solutions of the flow, we need to depend on special methods like high order compact (HOC) scheme, $(9,5)$ HOC formulation, fourth order streamfunction method. All these methods fall under the category of finite difference methods. However, the finite difference scheme proposed in this work gives more accurate numerical solutions of the flow variables with consumption of less time by the CPU. This happens because we always obtain tridiagonal and symmetric matrix after the numerical discretization, whereas in the work presented in the relevant literature mentioned above, they obtained a non-tridiagonal and sparse matrix after the numerical discretization performed for the purpose of the numerical computations of the flow variables.

As described in the beginning of this introduction, 2-D unsteady incompressible viscous flow has enormous scope for industrial applications. Moreover, due to the advantage of our present numerical method over the other numerical methods as cited earlier, we have been motivated in undertaking the current study. Moreover, the importance of these applications can be investigated only by determining numerical solutions of the unknown flow variables, streamfunction $\psi$, vorticityfunction $\xi$ for low Reynolds numbers $R e \leq 50$. To achieve this, we present, numerically, an investigation of the problem of 2-D unsteady incompressible flow in a driven square cavity using the streamfunction-vorticity $(\psi-\xi)$ formulation.

The main target of this work is to suitably use streamfunction-vorticity formulation to investigate the problem of 2-D unsteady viscous incompressible flow in a driven square cavity with moving top and bottom walls. AlternatingDirection-Implicit (ADI) scheme has been employed to discretize the vorticitytransport equation. We have proved the stability and convergence of the numerical scheme using matrix method. A general algorithm was used for this formulation in order to compute the numerical solutions of the flow variables for $R e \leq 50$.

The design of the current work is as follows: Section 2 provides the physical description, governing equations of the 2-D unsteady incompressible viscous flow in a driven cavity along with the initial and boundary conditions for a square cavity and streamfunction vorticity method. Section 3 describes numerical discretization of governing equations and specification of boundary 
conditions. Section 4 provides proof of the stability and convergence of the numerical scheme using the matrix method. Section 5 provides numerical computations and general algorithm for computation of numerical solution of the flow variables. Section 6 discusses the numerical results. Section 8 lays out the conclusions of the study.

\section{Problem Formulation}

\subsection{Physical description}

Figure 1 illustrates the geometry of the problem in this study along with the boundary conditions. ABCD is a square cavity in which a 2-D unsteady incompressible viscous flow is considered. Now, by sliding infinite long plates lying on top and bottom of the cavity, vorticity along the walls of the cavity is generated. Suppose that all variables are normalized so that the size of the cavity is $1 \times 1$ and the sliding velocities are 1 and -1 in the positive and negative $x$-directions respectively.

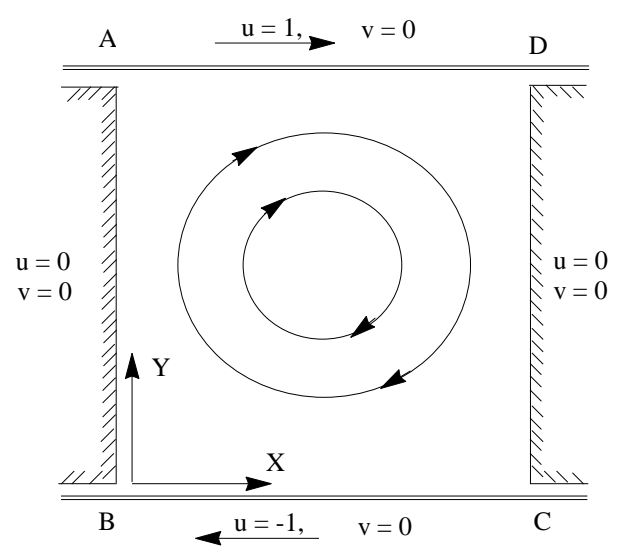

Figure 1. Square cavity flow caused by moving plates.

The boundary conditions are defined as no slip on the stationary walls (AB and $\mathrm{DC}$ ) and as slip on the moving walls (AD and $\mathrm{BC}$ ). We have assumed that, at all the four corner points of the square domain, the velocity components $(u, v)$ vanish. 


\subsection{Governing equations}

In the present investigation, unsteady 2-D incompressible flow in a driven square cavity with no-slip and slip wall boundary conditions has been considered. The governing equations of 2-D unsteady incompressible viscous flow are the continuity equation, and the two components of the momentum equations. Using the Boussinesq approximations, the dimensionless governing equations of this problem are expressed as follows:

$$
\begin{aligned}
\text { continuity equation } \quad \frac{\partial u}{\partial x} & +\frac{\partial v}{\partial y}=0 \\
x \text {-momentum equation } \quad \frac{\partial u}{\partial t} & +u \frac{\partial u}{\partial x}+v \frac{\partial u}{\partial y} \\
& =-\frac{\partial P}{\partial x}+\frac{1}{R e}\left(\frac{\partial^{2} u}{\partial x^{2}}+\frac{\partial^{2} u}{\partial y^{2}}\right) \\
y \text {-momentum equation } \quad \frac{\partial v}{\partial t} & +u \frac{\partial v}{\partial x}+v \frac{\partial v}{\partial y} \\
& =-\frac{\partial P}{\partial y}+\frac{1}{R e}\left(\frac{\partial^{2} v}{\partial x^{2}}+\frac{\partial^{2} v}{\partial y^{2}}\right),
\end{aligned}
$$

where $u, v, P$ and $R e$ are the velocity components along $x$ and $y$ axis, pressure and Reynolds number respectively. The initial, no-slip and slip wall boundary conditions are:

for $t=0$,

$$
u(x, y, 0)=0, \quad v(x, y, 0)=0,
$$

for $t>0$,

$$
\left.\begin{array}{ll}
\text { on plate } A B: & u=0, v=0 \\
\text { on plate } D C: & u=0, v=0 \\
\text { on plate } A D: & u=1, v=0 \\
\text { on plate } B C: & u=-1, v=0
\end{array}\right\} \text {. }
$$

\subsection{Streamfunction-Vorticity formulation}

To obtain the streamfunction-vorticity $(\psi-\xi)$ equation, pressure $P$ is eliminated from equation (2) and equation (3) by differentiating equation (2) with respect to $y$ and equation (3) with respect to $x$ and subtracting one from the other. 
The resulting equation is expressed with vorticity $\xi$ as the dependent variable which is defined by

$$
\xi=\frac{\partial v}{\partial x}-\frac{\partial u}{\partial y} .
$$

The result is

$$
\frac{\partial \xi}{\partial t}+u \frac{\partial \xi}{\partial x}+v \frac{\partial \xi}{\partial y}=\frac{1}{R e}\left(\frac{\partial^{2} \xi}{\partial x^{2}}+\frac{\partial^{2} \xi}{\partial y^{2}}\right) .
$$

Equation (7) is a parabolic PDE called vorticity-transport equation expressed in compact form as follows:

$$
\frac{D \xi}{D t}=\frac{1}{R e} \nabla^{2} \xi
$$

Now, define a streamfunction $\psi$ as

$$
\frac{\partial \psi}{\partial x}=-v, \frac{\partial \psi}{\partial y}=u .
$$

$\psi$ identically, satisfies the continuity equation (1). Further, use of definition of $\xi$ leads to

$$
\frac{\partial^{2} \psi}{\partial x^{2}}+\frac{\partial^{2} \psi}{\partial y^{2}}=-\xi
$$

or

$$
\nabla^{2} \psi=-\xi
$$

This is an elliptic PDE called Poisson equation.

\section{Numerical Discretization}

Discretization of the governing equations by finite difference method although a well-known technique we have adopted this technique in the present study due to its compatibility with the regularly shaped geometry, flow in a square cavity caused by moving plates. With the help of streamfunction-vorticity $(\psi-\xi)$ formulation as discussed above, the governing equations (1) to (3) of 2-D unsteady incompressible viscous flow in dimensionless form are expressed as: 


$$
\begin{aligned}
& \frac{\partial^{2} \psi}{\partial x^{2}}+\frac{\partial^{2} \psi}{\partial y^{2}}=-\xi \\
& \frac{\partial \xi}{\partial t}=\frac{1}{R e}\left(\frac{\partial^{2} \xi}{\partial x^{2}}+\frac{\partial^{2} \xi}{\partial y^{2}}\right)+\left(\frac{\partial \psi}{\partial x}\right)\left(\frac{\partial \xi}{\partial y}\right)-\left(\frac{\partial \psi}{\partial y}\right)\left(\frac{\partial \xi}{\partial x}\right)
\end{aligned}
$$

where $R e$ is the Reynolds number, $x$ and $y$ are the cartesian co-ordinates. Essentially, the system is composed of the Poisson equation for streamfunction (12) and the vorticity-transport equation (13).

To obtain the numerical solutions, the coupled equations (12) and (13) need to be solved in an iterative manner. There is a distinct advantages in using a scheme that will allow equation (13) to be solved by means of tridiagonal forms only. Therefore, a fully implicit scheme, namely Alternating-Direction-Implicit (ADI) scheme is used for solving numerically these coupled partial differential equations (12) and (13).

Consider a square numerical grid of size $1 \times 1$ having $n$ horizontal interior grid lines and an equal number of vertical grid lines as shown in Figure 2. Using the ADI scheme [5, pp.883] and applying the forward-time and centralspace (FTCS) finite difference quotients for the first and second order partial derivatives of $\psi$ and $\xi$ with respect to time and space variables in equations (12) and (13) as given below:

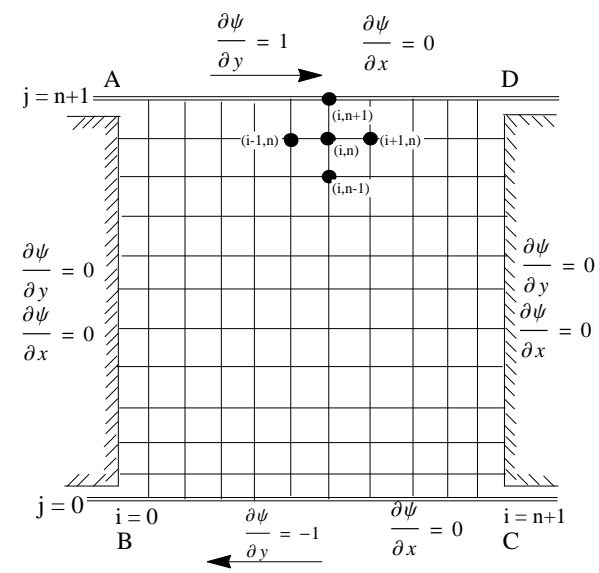

Figure 2. Finite difference grid of a square cavity flow caused by moving plates. 


$$
\begin{array}{rlrl}
\left.\frac{\partial^{2} \xi}{\partial x^{2}}\right|_{t} & =\frac{\xi_{i+1, j}^{t}-2 \xi_{i, j}^{t}+\xi_{i-1, j}^{t}}{\Delta x^{2}}, & \left.\frac{\partial \psi}{\partial x}\right|_{t}=\frac{\psi_{i+1, j}^{t}-\psi_{i-1, j}^{t}}{2 \Delta x}, \\
\left.\frac{\partial^{2} \xi}{\partial y^{2}}\right|_{t+\frac{1}{2}} & =\frac{\xi_{i, j+1}^{t+\frac{1}{2}}-2 \xi_{i, j}^{t+\frac{1}{2}}+\xi_{i, j-1}^{t+\frac{1}{2}}}{\Delta y^{2}}, & \left.\frac{\partial \psi}{\partial y}\right|_{t}=\frac{\psi_{i, j+1}^{t}-\psi_{i, j-1}^{t}}{2 \Delta y}, \\
\left.\frac{\partial \xi}{\partial x}\right|_{t}=\frac{\xi_{i+1, j}^{t}-\xi_{i-1, j}^{t}}{2 \Delta x}, & \left.\frac{\partial^{2} \psi}{\partial x^{2}}\right|_{t}=\frac{\psi_{i+1, j}^{t}-2 \psi_{i, j}^{t}+\psi_{i-1, j}^{t}}{\Delta x^{2}} \\
\left.\frac{\partial \xi}{\partial y}\right|_{t+\frac{1}{2}} & =\frac{\xi_{i, j+1}^{t+\frac{1}{2}}-\xi_{i, j-1}^{t+\frac{1}{2}}}{2 \Delta y}, & \left.\frac{\partial^{2} \psi}{\partial y^{2}}\right|_{t}=\frac{\psi_{i, j+1}^{t}-2 \psi_{i, j}^{t}+\psi_{i, j-1}^{t}}{\Delta y^{2}} \\
\left.\frac{\partial \xi}{\partial t}\right|_{t} & =\frac{\xi_{i, j}^{t+\frac{1}{2}}-\xi_{i, j}^{t}}{\frac{\Delta t}{2}}
\end{array}
$$

The discretized Poisson equation (12) for $\psi$ can be written as

$$
\frac{\psi_{i+1, j}^{t+1}-2 \psi_{i, j}^{t+1}+\psi_{i-1, j}^{t+1}}{\Delta x^{2}}+\frac{\psi_{i, j+1}^{t+1}-2 \psi_{i, j}^{t+1}+\psi_{i, j-1}^{t+1}}{\Delta y^{2}}=-\xi_{i, j}^{t+1}
$$

We choose $\Delta x=\Delta y$ so that the above discretized equation reduces to

$$
\psi_{i+1, j}^{t+1}+\psi_{i-1, j}^{t+1}+\psi_{i, j+1}^{t+1}+\psi_{i, j-1}^{t+1}-4 \psi_{i, j}^{t+1}=-\xi_{i, j}^{t+1} \Delta x^{2}
$$

The discretized vorticity-transport equation (13) over time step $t+\frac{1}{2}$ can be written as

$$
\begin{aligned}
\frac{\xi_{i, j}^{t+\frac{1}{2}}-\xi_{i, j}^{t}}{\Delta t}= & \frac{1}{2 R e}\left(\frac{\xi_{i+1, j}^{t}-2 \xi_{i, j}^{t}+\xi_{i-1, j}^{t}}{\Delta x^{2}}\right) \\
& -\frac{1}{2}\left(\frac{\partial \psi}{\partial y}\right)^{t}\left[\frac{\xi_{i+1, j}^{t}-\xi_{i-1, j}^{t}}{2 \Delta x}\right] \\
& +\frac{1}{2}\left(\frac{\partial \psi}{\partial x}\right)^{t}\left[\frac{\xi_{i, j+1}^{t+\frac{1}{2}}-\xi_{i, j-1}^{t+\frac{1}{2}}}{2 \Delta y}\right] \\
& +\frac{1}{2 R e}\left[\frac{\xi_{i, j+1}^{t+\frac{1}{2}}-2 \xi_{i, j}^{t+\frac{1}{2}}+\xi_{i, j-1}^{t+\frac{1}{2}}}{\Delta y^{2}}\right]
\end{aligned}
$$


The above equation becomes

$$
\begin{gathered}
{\left[\frac{\Delta t}{4 \Delta y}\left(\frac{\psi_{i+1, j}^{t}-\psi_{i-1, j}^{t}}{2 \Delta x}\right)-\frac{\Delta t}{2 R e \Delta y^{2}}\right] \xi_{i, j-1}^{t+\frac{1}{2}}+\left(1+\frac{\Delta t}{\operatorname{Re} \Delta y^{2}}\right) \xi_{i, j}^{t+\frac{1}{2}}} \\
+\left[-\frac{\Delta t}{4 \Delta y}\left(\frac{\psi_{i+1, j}^{t}-\psi_{i-1, j}^{t}}{2 \Delta x}\right)-\frac{\Delta t}{2 R e \Delta y^{2}}\right] \xi_{i, j+1}^{t+\frac{1}{2}} \\
=\xi_{i, j}^{t}+\frac{\Delta t}{2 \operatorname{Re} \Delta x^{2}}\left[\xi_{i+1, j}^{t}-2 \xi_{i, j}^{t}+\xi_{i-1, j}^{t}\right] \\
\quad-\frac{\Delta t}{8 \Delta x \Delta y}\left[\xi_{i+1, j}^{t}-\xi_{i-1, j}^{t}\right]\left[\psi_{i, j+1}^{t}-\psi_{i, j-1}^{t}\right] .
\end{gathered}
$$

This is the final discretized equation of (13) at time step $t+\frac{1}{2}$. Now, discretizing the vorticity-transport equation (13) over time step $t+1$ by introducing the finite difference quotients as described below:

$$
\begin{aligned}
\left.\frac{\partial^{2} \xi}{\partial x^{2}}\right|_{t+1} & =\frac{\xi_{i+1, j}^{t+1}-2 \xi_{i, j}^{t+1}+\xi_{i-1, j}^{t+1}}{\Delta x^{2}} \\
\left.\frac{\partial^{2} \xi}{\partial y^{2}}\right|_{t+\frac{1}{2}} & =\frac{\xi_{i, j+1}^{t+\frac{1}{2}}-2 \xi_{i, j}^{t+\frac{1}{2}}+\xi_{i, j-1}^{t+\frac{1}{2}}}{\Delta y^{2}} \\
\left.\frac{\partial \xi}{\partial x}\right|_{t+1} & =\frac{\xi_{i+1, j}^{t+1}-\xi_{i-1, j}^{t+1}}{2 \Delta x} \\
\left.\frac{\partial \xi}{\partial y}\right|_{t+\frac{1}{2}} & =\frac{\xi_{i, j+1}^{t+\frac{1}{2}}-\xi_{i, j-1}^{t+\frac{1}{2}}}{2 \Delta y} \\
\left.\frac{\partial \xi}{\partial t}\right|_{t+\frac{1}{2}} & =\frac{\xi_{i, j}^{t+1}-\xi_{i, j}^{t+\frac{1}{2}}}{\left(\frac{\Delta t}{2}\right)}
\end{aligned}
$$


Using the above equations into equation (13), we get

$$
\begin{aligned}
\xi_{i, j}^{t+1}-\xi_{i, j}^{t+\frac{1}{2}}= & \frac{\Delta t}{2 R e}\left[\frac{\xi_{i+1, j}^{t+1}-2 \xi_{i, j}^{t+1}+\xi_{i-1, j}^{t+1}}{\Delta x^{2}}\right] \\
& +\frac{\Delta t}{2 R e}\left[\frac{\xi_{i, j}^{t+\frac{1}{2}}-2 \xi_{i, j}^{t+\frac{1}{2}}+\xi_{i, j-1}^{t+\frac{1}{2}}}{\Delta y^{2}}\right] \\
& +\frac{\Delta t}{2}\left(\frac{\partial \psi}{\partial x}\right)^{t}\left[\frac{\xi_{i, j+1}^{t+\frac{1}{2}}-\xi_{i, j-1}^{t+\frac{1}{2}}}{2 \Delta y}\right] \\
& -\frac{\Delta t}{2}\left(\frac{\partial \psi}{\partial y}\right)^{t}\left[\frac{\xi_{i+1, j}^{t+1}-\xi_{i-1, j}^{t+1}}{2 \Delta x}\right] .
\end{aligned}
$$

This implies that

$$
\begin{gathered}
{\left[-\frac{\Delta t}{2 R e \Delta x^{2}}-\frac{\Delta t}{8 \Delta x \Delta y}\left(\psi_{i, j+1}^{t}-\psi_{i, j-1}^{t}\right)\right] \xi_{i-1, j}^{t+1}+\left(1+\frac{\Delta t}{R e \Delta x^{2}}\right) \xi_{i, j}^{t+1}} \\
+\left[-\frac{\Delta t}{2 R e \Delta x^{2}}+\frac{\Delta t}{8 \Delta x \Delta y}\left(\psi_{i, j+1}^{t}-\psi_{i, j-1}^{t}\right)\right] \xi_{i+1, j}^{t+1} \\
=\xi_{i, j}^{t+\frac{1}{2}}+\frac{\Delta t}{2 R e \Delta y^{2}}\left[\xi_{i, j+1}^{t+\frac{1}{2}}-2 \xi_{i, j}^{t+\frac{1}{2}}+\xi_{i, j-1}^{t+\frac{1}{2}}\right] \\
\quad+\frac{\Delta t}{8 \Delta y \Delta x}\left(\psi_{i+1, j}^{t}-\psi_{i-1, j}^{t}\right)\left[\xi_{i, j+1}^{t+\frac{1}{2}}-\xi_{i, j-1}^{t+\frac{1}{2}}\right]
\end{gathered}
$$

This is the final discretized equation of (13) at time step $t+1$.

\subsection{Specification of boundary conditions}

On the left plate AB: $\quad \xi_{0, j}=\frac{2\left(\psi_{0, j}-\psi_{1, j}+\left.\frac{\partial \psi}{\partial x}\right|_{0, j} \Delta x\right)}{\Delta x^{2}}$

On the right plate DC: $\quad \xi_{n+1, j}=\frac{2\left(\psi_{n+1, j}-\psi_{n, j}-\left.\frac{\partial \psi}{\partial x}\right|_{n+1, j} \Delta x\right)}{\Delta x^{2}}$

On the top plate AD: $\quad \xi_{i, n+1}=\frac{2\left(\psi_{i, n+1}-\psi_{i, n}-\left.\frac{\partial \psi}{\partial y}\right|_{i, n+1} \Delta y\right)}{\Delta y^{2}}$

On the bottom plate BC: $\xi_{i, 0}=\frac{2\left(\psi_{i, 0}-\psi_{i, 1}+\left.\frac{\partial \psi}{\partial y}\right|_{i, 0} \Delta y\right)}{\Delta y^{2}}$. 


\section{Stability and Convergence of the Numerical Scheme}

In this section, we will prove the stability and convergence of the numerical (ADI) method used in the numerical discretization based on the criteria proposed by Peaceman and Rachford [26]. We now introduce the finite-difference formulae for first and second-order partial derivatives of $\xi$ with respect to $x$ and $y$ as proposed in [19, pp. 94], which are given by

$$
\begin{aligned}
& D_{+, x}\left(h_{x}\right) \xi_{i, j}=\frac{\xi_{i+1, j}-\xi_{i, j}}{h_{x}}+O\left(h_{x}\right), \\
& D_{-, x}\left(h_{x}\right) \xi_{i, j}=\frac{\xi_{i, j}-\xi_{i-1, j}}{h_{x}}+O\left(h_{x}\right), \\
& D_{0, x}\left(h_{x}\right) \xi_{i, j}=\frac{\xi_{i+1, j}-\xi_{i-1, j}}{2 h_{x}}+O\left(h_{x}^{2}\right) .
\end{aligned}
$$

Similarly, for second-order partial derivatives we will employ the second central difference,

$$
D_{0, x}^{2}\left(h_{x}\right) \xi_{i, j}=\frac{\xi_{i+1, j}-2 \xi_{i, j}+\xi_{i-1, j}}{h_{x}^{2}}+O\left(h_{x}^{2}\right),
$$

where $h_{x}$ is the grid spacing in $x$-direction. We adopt similar formulae in $y$ direction. Let $k=\Delta t$ denotes the grid spacing in time $(t)$-direction. Further, we assume $A=D_{0, x}^{2}, B=D_{0, y}^{2}, C=D_{0, x}$ and $D=D_{0, y}$. Now, discretizing the equation (13) at time step $t+\frac{1}{2}$ we get

$$
\xi_{i, j}^{t+\frac{1}{2}}-\xi_{i, j}^{t}=\frac{k}{2 R e} A \xi_{i, j}^{t}-\frac{k}{2} u_{i, j} C \xi_{i, j}^{t}+\frac{k}{2 R e} B \xi_{i, j}^{t+\frac{1}{2}}-\frac{k}{2} v_{i, j} D \xi_{i, j}^{t+\frac{1}{2}},
$$

which gives

$$
\left[I+\frac{k}{2} v_{i, j} D-\frac{k}{2 R e} B\right] \xi_{i, j}^{t+\frac{1}{2}}=\left[I-\frac{k}{2} u_{i, j} C+\frac{k}{2 R e} A\right] \xi_{i, j}^{t} .
$$

Now, discretizing the equation (13) at time step $t+1$ we get

$$
\xi_{i, j}^{t+1}-\xi_{i, j}^{t+\frac{1}{2}}=\frac{k}{2 R e} A \xi_{i, j}^{t+1}-\frac{k}{2} u_{i, j} C \xi_{i, j}^{t+1}+\frac{k}{2 R e} B \xi_{i, j}^{t+\frac{1}{2}}-\frac{k}{2} v_{i, j} D \xi_{i, j}^{t+\frac{1}{2}},
$$

which gives

$$
\left[I+\frac{k}{2} u_{i, j} C-\frac{k}{2 R e} A\right] \xi_{i, j}^{t+1}=\left[I-\frac{k}{2} v_{i, j} D+\frac{k}{2 R e} B\right] \xi_{i, j}^{t+\frac{1}{2}} .
$$

Now, equations (23) and (24) together give 


$$
\begin{aligned}
{\left[I+\frac{k}{2} u_{i, j} C-\frac{k}{2 R e} A\right] \xi_{i, j}^{t+1}=} & {\left[I-\frac{k}{2} v_{i, j} D+\frac{k}{2 R e} B\right] } \\
& \times\left[I+\frac{k}{2} v_{i, j} D-\frac{k}{2 R e} B\right]^{-1} \\
& \times\left[I-\frac{k}{2} u_{i, j} C+\frac{k}{2 R e} A\right] \xi_{i, j}^{t} .
\end{aligned}
$$

Rewriting the equations (23), (24) and (25) we obtain the following form:

$$
\begin{aligned}
\xi_{i, j}^{t+\frac{1}{2}} & =E \xi_{i, j}^{t}, \\
\xi_{i, j}^{t+1} & =F \xi_{i, j}^{t+\frac{1}{2}}, \\
\xi_{i, j}^{t+1} & =F E \xi_{i, j}^{t},
\end{aligned}
$$

where

$$
\begin{aligned}
& E=\left[I+\frac{k}{2} v_{i, j} D-\frac{k}{2 R e} B\right]^{-1}\left[I-\frac{k}{2} u_{i, j} C+\frac{k}{2 R e} A\right], \\
& F=\left[I+\frac{k}{2} u_{i, j} C-\frac{k}{2 R e} A\right]^{-1}\left[I-\frac{k}{2} v_{i, j} D+\frac{k}{2 R e} B\right] .
\end{aligned}
$$

In terms of a matrix stability analysis, it is required that the spectral radius of $E$ be less than or equal to unity, i.e. $\rho(E) \leq 1$, for the first time split step to be stable [19, pp.94]. Similarly, we require $\rho(F) \leq 1$, for the second time split step to be stable.

Now recall that for any matrix $M, \rho(M) \leq\|M\|$ for all matrix norms $\|\cdot\|$. Also recall that if we define

$$
z_{o}^{t} \equiv v_{o}^{t}-u_{o}^{t}
$$

where $v_{o}^{t}$ is the exact solution of the differential equation and $u_{o}^{t}$ is the computed one, we have

$$
z_{o}^{t+1}=F E z_{o}^{t} .
$$

Thus,

$$
\left\|z_{o}^{t+1}\right\|=\left\|F E z_{o}^{t}\right\| \leq\|F\|\|E\|\left\|z_{o}^{t}\right\| .
$$

If we now take $\|\cdot\|$ to be the spectral norm for the matrices (and 2-norm for vectors) so $\|\cdot\|=\rho(\cdot)$, which is valid provided $E$ and $F$ are diagonalizable with real eigenvalues, we have 


$$
\left\|z_{o}^{t+1}\right\| \leq \rho(F) \rho(E)\left\|z_{o}^{t}\right\| .
$$

Thus, our task reduces to show that $\rho(E) \leq 1$ and $\rho(F) \leq 1$ to achieve

$$
\left\|z_{o}^{t+1}\right\| \leq\left\|z_{o}^{t}\right\|,
$$

which proves the unconditional stability of the method. Consider equations (26) and (27) with $A=B$ and $C=D$. We note that this occurs for the vorticity transport equation if the same spatial step size is used in both directions. Now, writing the discretized equation of (26), we get

$$
\begin{aligned}
& {\left[\frac{\Delta t}{4 \Delta y} v_{i, j}-\frac{\Delta t}{2 R e \Delta y^{2}}\right] \xi_{i, j+1}^{t+\frac{1}{2}}+\left(1+\frac{\Delta t}{R e \Delta y^{2}}\right) \xi_{i, j}^{t+\frac{1}{2}}} \\
& +\left[-\frac{\Delta t}{4 \Delta y} v_{i, j}-\frac{\Delta t}{2 R e \Delta y^{2}}\right] \xi_{i, j-1}^{t+\frac{1}{2}} \\
& =\left[-\frac{\Delta t}{4 \Delta x} u_{i, j}+\frac{\Delta t}{2 R e \Delta x^{2}}\right] \xi_{i, j+1}^{t}+\left(1-\frac{\Delta t}{R e \Delta x^{2}}\right) \xi_{i, j}^{t} \\
& \quad+\left[\frac{\Delta t}{4 \Delta x} u_{i, j}+\frac{\Delta t}{2 R e \Delta x^{2}}\right] \xi_{i, j-1}^{t} .
\end{aligned}
$$

Now, varying the values for nodes in a domain consisting of $n \times n$ interior grid points, we obtain a system of equations. Writing these system of equations in matrix form, we obtained the following form:

$$
\left[\begin{array}{ccccc}
a & b & & & \\
c & a & b & & \\
& \cdot & \cdot & \cdot & \\
& & c & a & b \\
& & & c & a
\end{array}\right]\left[\begin{array}{c}
\xi_{i, j_{1}}^{t+\frac{1}{2}} \\
\xi_{i, j_{2}}^{t+\frac{1}{2}} \\
\vdots \\
\xi_{i, j_{n}}^{t+\frac{1}{2}}
\end{array}\right]=\left[\begin{array}{ccccc}
a^{\prime} & b^{\prime} & & & \\
c^{\prime} & a^{\prime} & b^{\prime} & & \\
& \cdot & \cdot & \cdot & \\
& & c^{\prime} & a^{\prime} & b^{\prime} \\
& & & c^{\prime} & a^{\prime}
\end{array}\right]\left[\begin{array}{c}
\xi_{i, j_{1}}^{t} \\
\xi_{i, j_{2}}^{t} \\
\vdots \\
\xi_{i, j_{n}}^{t}
\end{array}\right]+b_{j}
$$

where

$$
\begin{aligned}
a & =\left[1+\frac{\triangle t}{R e \triangle y^{2}}\right], b=\left[\frac{\triangle t}{4 \triangle y} v_{i, j}-\frac{\triangle t}{2 R e \triangle y^{2}}\right], \\
c & =\left[-\frac{\triangle t}{4 \triangle y} v_{i, j}-\frac{\triangle t}{2 R e \triangle y^{2}}\right], \\
a^{\prime} & =\left[1-\frac{\triangle t}{\operatorname{Re} \triangle x^{2}}\right], b^{\prime}=\left[-\frac{\triangle t}{4 \triangle x} u_{i, j}+\frac{\triangle t}{2 R e \triangle x^{2}}\right], \\
c^{\prime} & =\left[\frac{\triangle t}{4 \triangle x} u_{i, j}+\frac{\triangle t}{2 \operatorname{Re} \triangle x^{2}}\right],
\end{aligned}
$$


and $b_{j}$ is a column vector of known boundary values of $\xi_{i, j_{0}}^{t+\frac{1}{2}}, \xi_{i, j_{n+1}}^{t+\frac{1}{2}}$ at time $t+\frac{1}{2}$, and zeros. Rewriting the above system as follows

$$
\mathcal{P}_{1} \zeta^{t+\frac{1}{2}}=\mathcal{Q}_{1} \zeta^{t}+\mathcal{R}_{1}^{t+\frac{1}{2}},
$$

where the matrices $\mathcal{P}_{1}, \mathcal{Q}_{1}$ are of order $n$ as shown above, $\zeta^{t+\frac{1}{2}}$ and $\zeta^{t}$ denote the column vectors with components

$$
\xi_{i, j_{1}}^{t+\frac{1}{2}}, \xi_{i, j_{2}}^{t+\frac{1}{2}}, \ldots \xi_{i, j_{n}}^{t+\frac{1}{2}}, \quad \text { and } \quad \xi_{i, j_{1}}^{t}, \xi_{i, j_{2}}^{t}, \ldots \xi_{i, j_{n}}^{t}
$$

respectively, $\mathcal{R}_{1}^{t+\frac{1}{2}}$ denotes the column vector of $\xi_{i, j_{0}}^{t+\frac{1}{2}}, \xi_{i, j_{n+1}}^{t+\frac{1}{2}}$ at time $t+\frac{1}{2}$, and zeros. Now, calculating the eigenvalues of matrix $\mathcal{P}_{1}$ in the above matrix form,

$$
\begin{aligned}
b c & =\left[\frac{\triangle t}{4 \triangle y} v_{i, j}-\frac{\Delta t}{2 R e \triangle y^{2}}\right]\left[-\frac{\Delta t}{4 \triangle y} v_{i, j}-\frac{\Delta t}{2 R e \triangle y^{2}}\right] \\
& =\frac{\Delta t^{2}}{4 R e^{2} \Delta y^{4}}\left[1-\frac{v_{i, j}^{2} \Delta y^{2} R e^{2}}{4}\right] .
\end{aligned}
$$

So, the eigenvalues of matrix $\mathcal{P}_{1}$ are

$$
\begin{aligned}
\lambda_{p s}=( & \left.1+\frac{\Delta t}{R e \Delta y^{2}}\right)+\frac{\Delta t}{R e \Delta y^{2}}\left(\sqrt{1-\frac{v_{i, j}^{2} \Delta y^{2} R e^{2}}{4}}\right) \\
& \times \cos \left(\frac{s \pi}{n+1}\right), \text { where } s=1,2, \ldots, n .
\end{aligned}
$$

Now, calculating the eigenvalues of matrix $\mathcal{Q}_{1}$ in above system,

$$
\begin{gathered}
b^{\prime} c^{\prime}=\left[-\frac{\triangle t}{4 \triangle x} u_{i, j}+\frac{\triangle t}{2 R e \triangle x^{2}}\right]\left[\frac{\triangle t}{4 \triangle x} u_{i, j}+\frac{\triangle t}{2 R e \triangle x^{2}}\right] \\
=\frac{\Delta t^{2}}{4 R e^{2} \Delta x^{4}}\left[1-\frac{u_{i, j}^{2} \Delta x^{2} R e^{2}}{4}\right] .
\end{gathered}
$$

Therefore, the eigenvalues of matrix $\mathcal{Q}_{1}$ are

$$
\begin{aligned}
\lambda_{q s}= & \left(1-\frac{\Delta t}{\operatorname{Re} \triangle x^{2}}\right)+\frac{\Delta t}{\operatorname{Re} \Delta x^{2}}\left(\sqrt{1-\frac{u_{i, j}^{2} \Delta x^{2} R e^{2}}{4}}\right) \\
& \times \cos \left(\frac{s \pi}{n+1}\right), \text { where } s=1,2, \ldots, n .
\end{aligned}
$$


Thus, for the stability we must have

$$
\begin{aligned}
& \left\|\mathcal{P}_{1}^{-1} \mathcal{Q}_{1}\right\|_{2}=\rho\left(\mathcal{P}_{1}^{-1} \mathcal{Q}_{1}\right)=\max _{s}\left|\frac{\lambda_{q s}}{\lambda_{p s}}\right| \leq 1, \quad s=1,2, \ldots, n . \\
& \left\|\mathcal{P}_{1}^{-1} \mathcal{Q}_{1}\right\|_{2}=\max _{s}\left|\frac{\left(1-\frac{\Delta t}{\operatorname{Re} \Delta x^{2}}\right)+\frac{\Delta t}{\operatorname{Re\Delta x^{2}}}\left(\sqrt{1-\frac{u_{i, j}^{2} \Delta x^{2} R e^{2}}{4}}\right) \cos \left(\frac{s \pi}{n+1}\right)}{\left(1+\frac{\Delta t}{R e \Delta y^{2}}\right)+\frac{\Delta t}{\operatorname{Re} \Delta y^{2}}\left(\sqrt{1-\frac{v_{i, j}^{2} \Delta y^{2} R e^{2}}{4}}\right) \cos \left(\frac{s \pi}{n+1}\right)}\right| \leq 1 .
\end{aligned}
$$

We have chosen

$$
\begin{array}{r}
\Delta x=\Delta y, r=\frac{\Delta t}{R e \Delta x^{2}}=\frac{\Delta t}{R e \Delta y^{2}}, \\
M=\frac{u_{i, j}^{2} \Delta x^{2} R e^{2}}{4} \text { and } M^{\prime}=\frac{v_{i, j}^{2} \Delta y^{2} R e^{2}}{4} .
\end{array}
$$

Then the equation (28) can be expressed in the form

$$
\left\|\mathcal{P}_{1}^{-1} \mathcal{Q}_{1}\right\|_{2}=\max _{s}\left|\frac{1-r+r \sqrt{1-M} \cos \left(\frac{s \pi}{n+1}\right)}{1+r+r \sqrt{1-M^{\prime}} \cos \left(\frac{s \pi}{n+1}\right)}\right| \leq 1, \quad s=1,2, \ldots, n .
$$

It is easy to observe that

$$
|M| \leq 1, \quad\left|M^{\prime}\right| \leq 1
$$

or,

$$
\left|\frac{u_{i, j}^{2} \Delta x^{2} R e^{2}}{4}\right| \leq 1, \quad\left|\frac{v_{i, j}^{2} \Delta y^{2} R e^{2}}{4}\right| \leq 1
$$

Thus, we have

$$
\left|u_{i, j} \Delta x R e\right| \leq 2
$$

and

$$
\left|v_{i, j} \Delta y R e\right| \leq 2
$$

Also letting

$$
f=\frac{1-r+r \sqrt{1-M} \cos \left(\frac{s \pi}{n+1}\right)}{1+r+r \sqrt{1-M^{\prime}} \cos \left(\frac{s \pi}{n+1}\right)}, \text { we see that } \frac{d f}{d s}=0 \text { gives } s=n+1 .
$$

For this value of $s,\left\|\mathcal{P}_{1}^{-1} \mathcal{Q}_{1}\right\|_{2}$ has maximum value, therefore equation (29) reduces to 


$$
\left|\frac{1-r-r \sqrt{1-M}}{1+r-r \sqrt{1-M^{\prime}}}\right| \leq 1 .
$$

The left side inequality implies

$$
-1-r+r \sqrt{1-M^{\prime}} \leq 1-r-r \sqrt{1-M},
$$

which gives

$$
r \leq \frac{2}{\left[\sqrt{1-M^{\prime}}+\sqrt{1-M}\right]}<1 .
$$

Since

$$
|M| \leq 1, \quad\left|M^{\prime}\right| \leq 1
$$

Also

$$
r=\frac{\Delta t}{R e \Delta x^{2}}=\frac{\Delta t}{R e \Delta y^{2}} \geq 0 .
$$

Thus, from equations (30) and (31) we have

$$
0 \leq r<1 \text {, }
$$

i.e.

$$
\Delta t<R e \Delta x^{2} .
$$

It is notable that the same result is obtained when we apply the above procedure to finite difference equation $(27)$ to show $\rho(D) \leq 1$. It should be clear from this development that since all that really is needed is $\rho(C) \cdot \rho(D) \leq 1$, it is possible for one or the other (but not both) individual split steps to be unstable while still maintaining overall stability of the complete method. Hence, the numerical scheme we have used is unconditionally stable. The scheme is consistent as the limiting value of the local truncation is zero as $\Delta t, \Delta x$ and $\Delta y \longrightarrow 0$. So, by Lax's equivalence theorem [3, pp. 72], the given scheme is convergent.

\section{Numerical Computations}

We obtained the numerical computation of the unknown flow variables $\psi, \xi$, $u, v$ for the present problem with the aid of a computer programme developed and run in $\mathrm{C}++$ compiler. The input data for the relevant parameters in the governing equations like Reynolds number $(R e)$ has been properly chosen incompatible with the present problem considered. 


\subsection{Algorithm for obtaining numerical solutions by streamfunction-vorticity formulation}

The algorithm for obtaining the numerical solutions by streamfunction-vorticity formulation consists of the following steps:

Step 1. Specify the initial values for $\xi$ and $\psi$ at time $t=0$.

Step 2. Solve the vorticity transport equation (8) for $\xi$ at each interior grid point at time $t+\Delta t$.

Step 3. Iterate for new $\psi$ values at all points by solving the Poisson equation (10) using new $\xi$ values at interior points.

Step 4. Find the velocity components $u=\frac{\partial \psi}{\partial y}$ and $v=-\frac{\partial \psi}{\partial x}$.

Step 5. Determine the values of $\xi$ on the boundaries using $\psi$ and $\xi$ values at interior points.

Step 6. Return to step 2 if the solution does not converge.

\section{Results and Discussion}

We used the Alternating-Direction-Implicit (ADI) scheme to carry out the numerical computations of the unknown flow variables $u, v, \psi, \xi$ for the present problem. We summarized this method under an algorithm for streamfunctionvorticity formulation as described under Section 5.1 above. We used a computer code developed and run in $\mathrm{C}++$ compiler to execute this. To verify our computer code, the numerical results obtained by the present method were compared with the benchmark results reported in [1]. It is seen that the results obtained in the present work are in good agreement with those reported in [1] at low Reynolds number $R e=100$. This indicates the validity of the numerical code that we developed. 


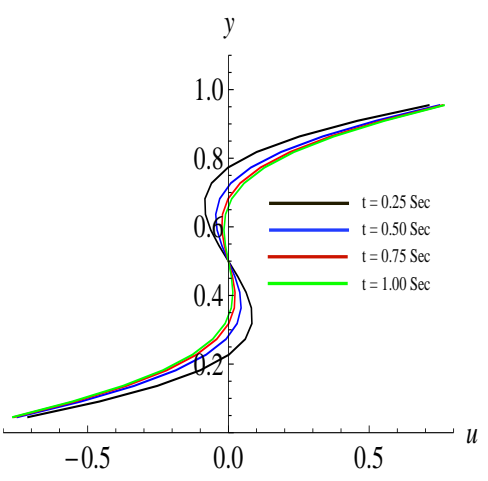

(a) $R e=15$

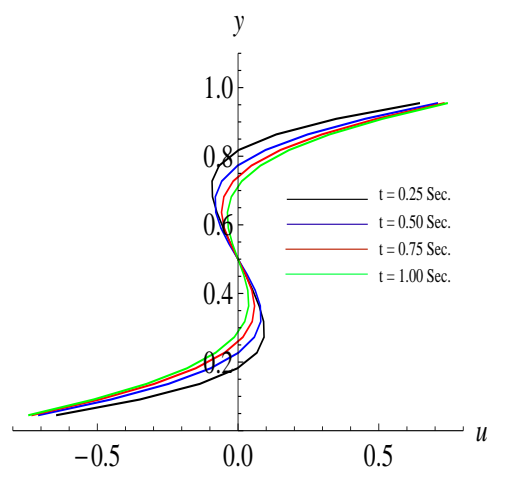

(b) $R e=30$

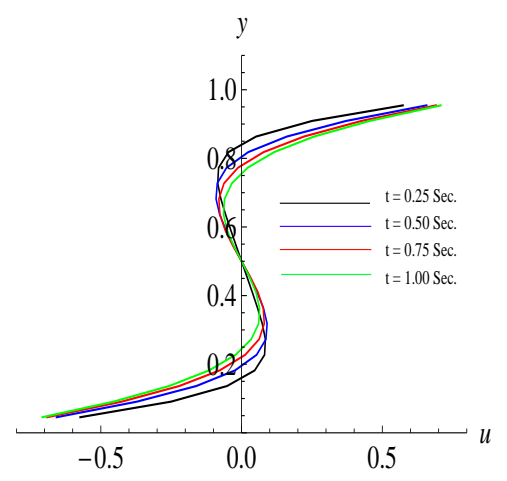

(c) $R e=50$

Figure 3. $u$-velocity profiles along the vertical line through geometric center of the square cavity for: (i) $R e=15$, (ii) $R e=30$, (iii) $R e=50$, at different time levels.

Figure 3 illustrates the variation of $u$-velocity along the vertical line through geometric center of the square cavity at different time levels for different Reynolds numbers $R e=15,30$ and 50. We observed that, the $u$-velocity is almost same near the top and bottom wall of the square cavity above and below the geometric center respectively for $R e=15$ and 30 . We also observed that, the absolute value of $u$-velocity first decrease, then increases, and finally, decreases in the vicinity of the top wall and the same behaviour is observed below the geometric center. 


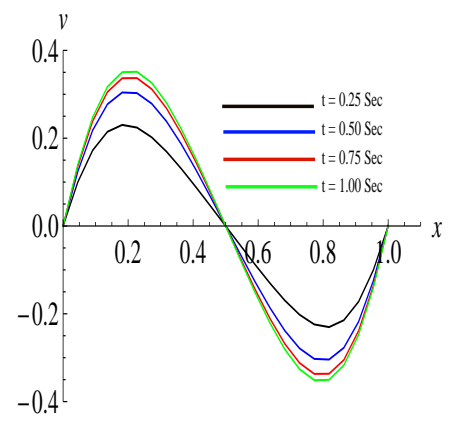

(a) $R e=15$

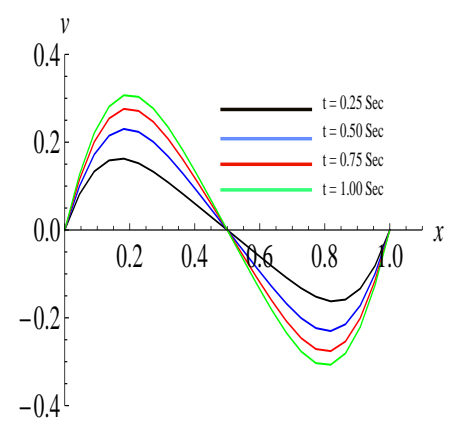

(b) $R e=30$

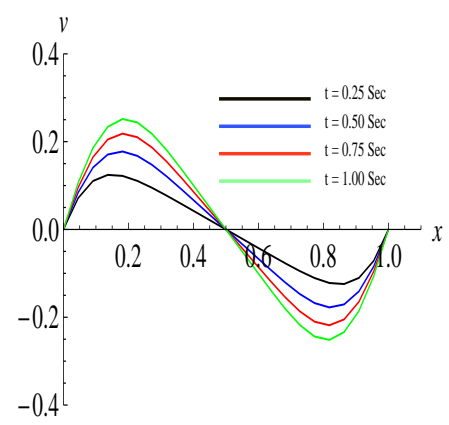

(c) $R e=50$

Figure 4. $v$-velocity profiles along the horizontal line through geometric center of the square cavity for: (i) $R e=15$ (ii) $R e=30$ (iii) $R e=50$, at different time levels.

Figure 4 illustrates the variation of $v$-velocity along the horizontal line through geometric center of the square cavity at different time levels for different Reynolds numbers $R e=15,30$ and 50. We observed that, the absolute value of $v$-velocity first increases uniformly, and finally, converges to the boundary of the right wall. The behaviour of $v$-velocity from the left side wall towards the geometric center of the cavity is that it increases uniformly, and converges at the geometric center of the cavity. We also observed that the absolute value of $v$-velocity increases uniformly as time increases, and converges at the geometric center, and the same behaviour is also observed to the right side of the geometric center for Reynolds numbers $R e=15,30$ and 50 . 

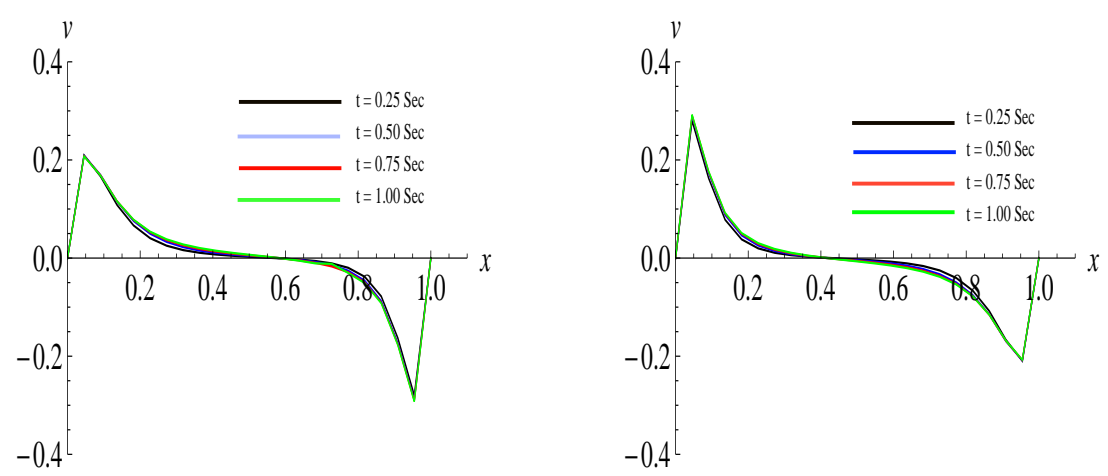

Figure 5. $v$-velocity profiles in the Figure 6. $v$-velocity profiles in the vicinity of moving top wall of the vicinity of moving bottom wall of square cavity for $R e=50$ at different the square cavity for $R e=50$ at diftime levels. ferent time levels.

Figures 5 and 6 illustrate the $v$-velocity profiles in the vicinity of moving top and bottom walls of the square cavity for $R e=50$ at different time levels. We observed that, the $v$-velocity in the vicinity of moving top wall of the square cavity attains a maximum value, and then, decreases toward the geometric center. It, then, increases. Moreover, for the moving bottom wall as well, we observed the same behaviour.

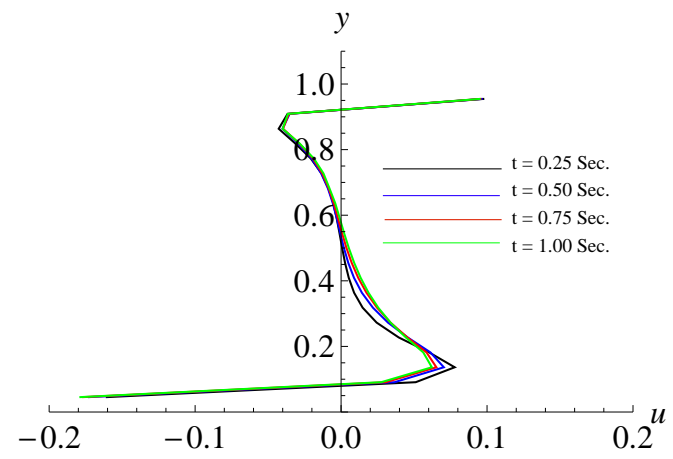

Figure 7. $u$-velocity profiles in the vicinity of left stationary wall of the square cavity for $R e=50$ at different time levels.

Figure 7 illustrates the $u$-velocity profiles in the vicinity of left stationary wall of the square cavity at different time levels for $R e=50$. We observed that, 
the $u$-velocity profiles near the left wall first increases uniformly, and finally, converges to the top boundary.

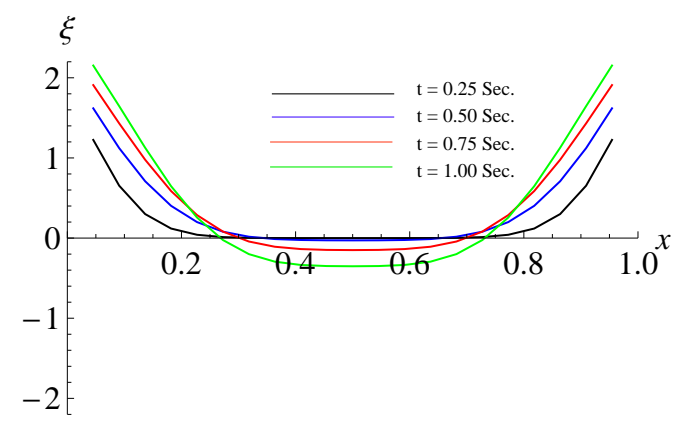

Figure 8. vorticity contours for $R e=50$ at different time levels.

Figure 8 illustrates the vorticity contours along the horizontal line through geometric center of the square cavity at different time levels for $R e=50$. We observed that, the vorticity contours decreased in between the left wall boundary to the midpoint of the square domain. It, then, increases in between the midpoint to the right wall boundary.

The streamline contours for different Reynolds numbers $R e=15,30$ and 50 for different time levels $(t=0.25 \mathrm{sec}, 0.50 \mathrm{sec}, 0.75 \mathrm{sec}, 1.00 \mathrm{sec})$ have been depicted in Figures 9, 10 and 11 respectively. We observed that, when the top wall is moving in the positive direction and the bottom wall is moving in the negative direction, two streamline contours are generated, one above the geometric center and the other below the geometric center in clock wise direction. We also observed that, the size of the streamline contours decreases with an increase of time. 


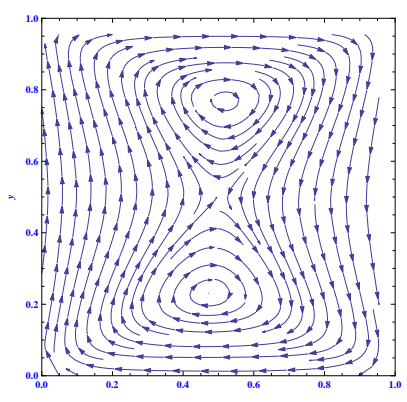

(a) $t=0.25 \mathrm{sec}$

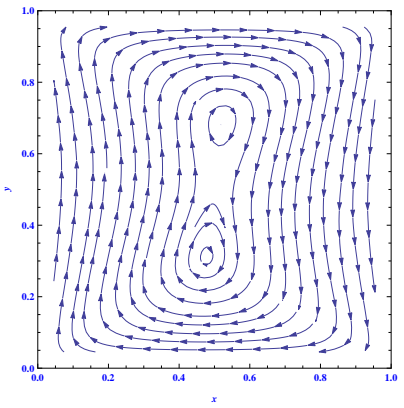

(c) $t=0.75 \mathrm{sec}$

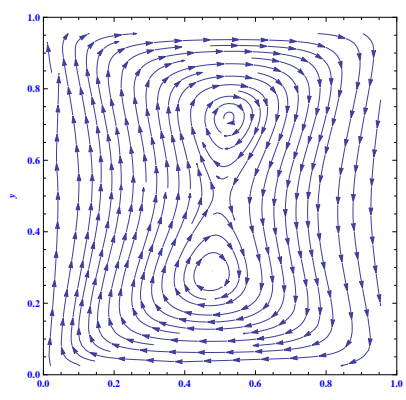

(b) $t=0.50 \mathrm{sec}$

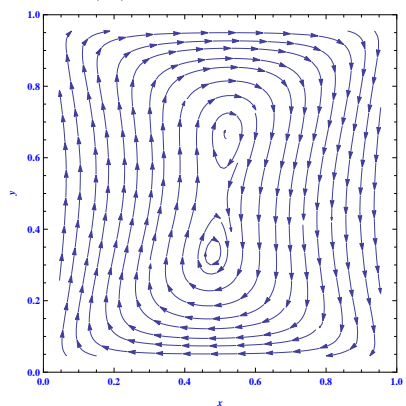

(d) $t=1.00 \mathrm{sec}$

Figure 9. Streamline contours for $R e=15$ at different time levels:(a) $\mathrm{t}$ $=0.25 \mathrm{sec} ;(\mathrm{b}) \mathrm{t}=0.50 \mathrm{sec} ;(\mathrm{c}) \mathrm{t}=0.75 \mathrm{sec} ;(\mathrm{d}) \mathrm{t}=1.00 \mathrm{sec}$. 


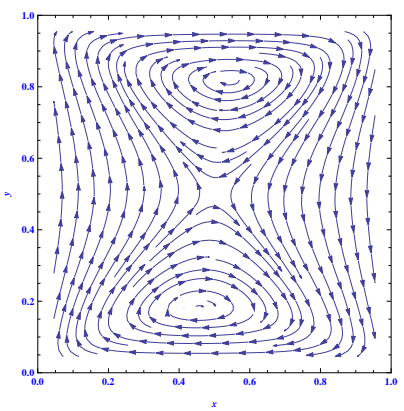

(a) $t=0.25 \mathrm{sec}$

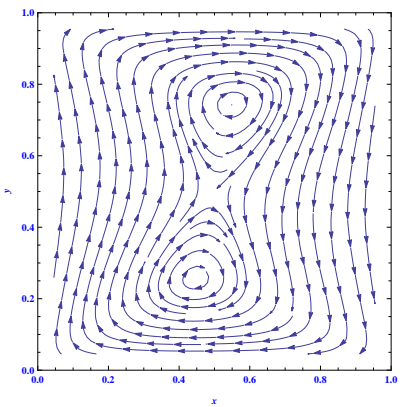

(c) $t=0.75 \mathrm{sec}$

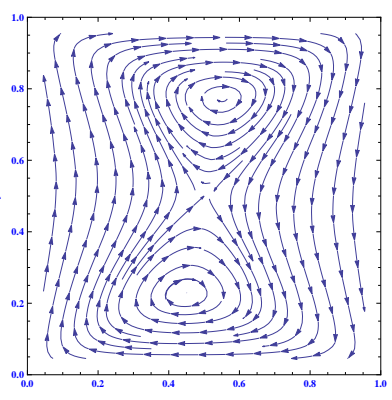

(b) $t=0.50 \mathrm{sec}$

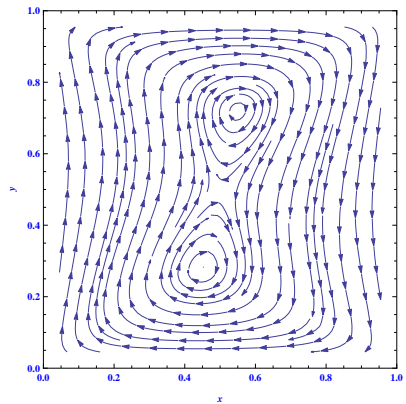

(d) $t=1.00 \mathrm{sec}$

Figure 10. Streamline contours for $R e=30$ at different time levels:(a) $\mathrm{t}$ $=0.25 \mathrm{sec} ;(\mathrm{b}) \mathrm{t}=0.50 \mathrm{sec} ;(\mathrm{c}) \mathrm{t}=0.75 \mathrm{sec} ;(\mathrm{d}) \mathrm{t}=1.00 \mathrm{sec}$. 


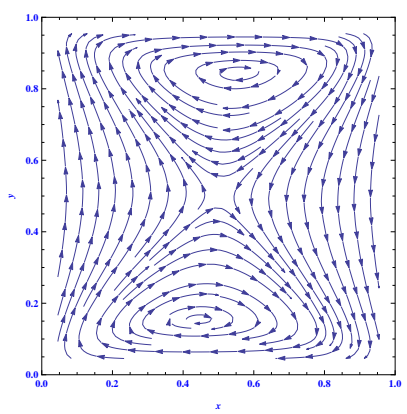

(a) $t=0.25 \mathrm{sec}$

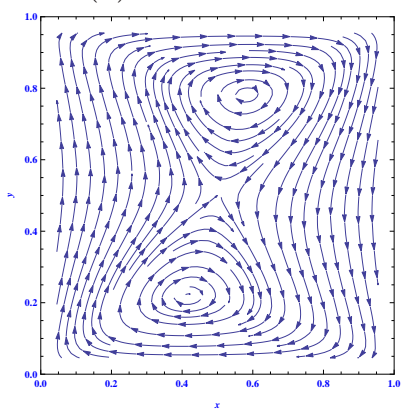

(c) $t=0.75 \mathrm{sec}$

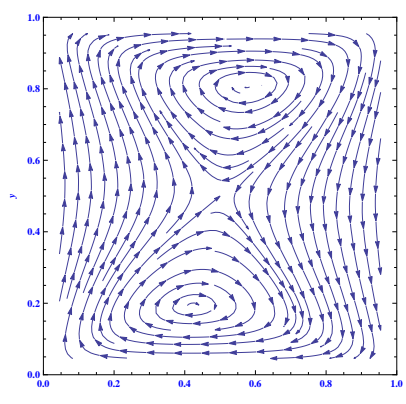

(b) $t=0.50 \mathrm{sec}$

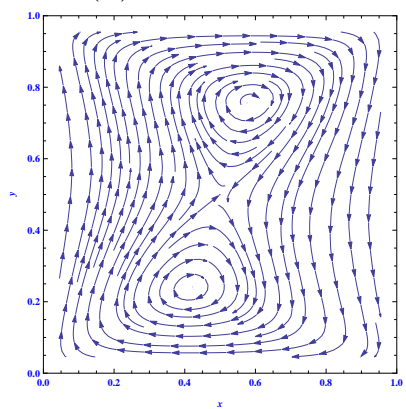

(d) $t=1.00 \mathrm{sec}$

Figure 11. Streamline contours for $R e=50$ at different time levels:(a) $\mathrm{t}$ $=0.25 \mathrm{sec} ;(\mathrm{b}) \mathrm{t}=0.50 \mathrm{sec} ;(\mathrm{c}) \mathrm{t}=0.75 \mathrm{sec} ;(\mathrm{d}) \mathrm{t}=1.00 \mathrm{sec}$.

\section{Code Validation}

To check the validity of our present computer code used to obtain the numerical results of $u$-velocity and $v$-velocity, we have compared our present results with those benchmark results given by Ghia et al. [1] and it has been found that they are in good agreement. 


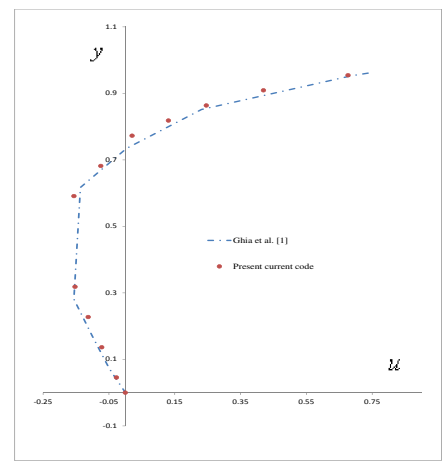

Figure 12. Comparison of the numerical results of $u$-velocity along the vertical line through geometric center of the square cavity for $R e=100$.

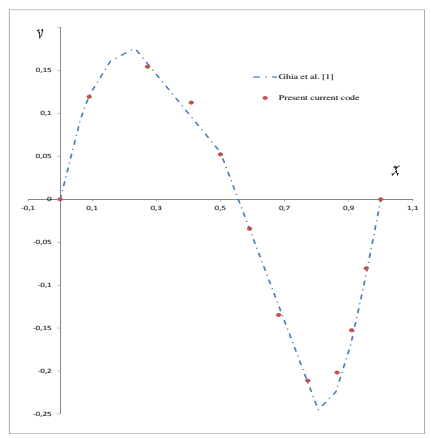

Figure 13. Comparison of the numerical results of $v$-velocity along the horizontal line through geometric center of the square cavity for $R e=100$.

\section{Conclusions}

In this study, we have obtained the numerical solutions for 2-D unsteady incompressible flow in a driven square cavity, along with no-slip and slip boundary conditions, using streamfunction-vorticity formulation. Using a general algorithm for this formulation, we carried out the numerical computations of the flow variables, streamfunction $\psi$ and vorticityfunction $\xi$ for low Reynolds numbers $R e \leq 50$. With the aid of numerical computations, we illustrate the variation of the $u$-velocity along the vertical line and $v$-velocity along the horizontal line through the geometric center of the square cavity. We observed that the $u$-velocity is almost same near the top and bottom wall of the square cavity above and below the geometric center respectively for $R e=15$ and 30 . We also observed that the absolute value of $u$-velocity first decreases, then increases, and finally, decreases in the vicinity of the top wall and the same behaviour is observed below the geometric center. We observed that the absolute value of the $v$-velocity increases uniformly as time level increases. However, as we move towards right of the geometric center, we observed the same behaviour at both Reynolds numbers $R e=30$ and 50. Furthermore, we found that the $v$-velocity 
at the right wall of the square cavity attains a maximum value, and then decreases towards the geometric center. It, then, increases. Figure 11 illustrates the $u$-velocity profiles at the left stationary wall of the square cavity.

Furthermore, we employed the current numerical computations to show that vorticity contours decreases in between left wall boundary to the midpoint of the domain. It, then, increases in between the midpoint to the right wall boundary. The numerical computations of the streamfunction for different low Reynolds numbers at different time levels, showed that two streamline contours are generated, one above the geometric center, and the other below the geometric center, in clockwise direction. We also observed that the size of the streamline contours decreased when the time level increased.

\section{Nomenclature}

Re Reynolds number

$x, y \quad$ cartesian co-ordinates

$\Delta x \quad$ grid spacing along $x$-axis

$\Delta y \quad$ grid spacing along $y$-axis

$\Delta t \quad$ time spacing

$u \quad x$-component of velocity

$v \quad y$-component of velocity

$u_{i, j}^{t} \quad x$-component of velocity at $(i, j)$ node at time $t$

$v_{i, j}^{t} \quad y$-component of velocity at $(i, j)$ node at time $t$ 


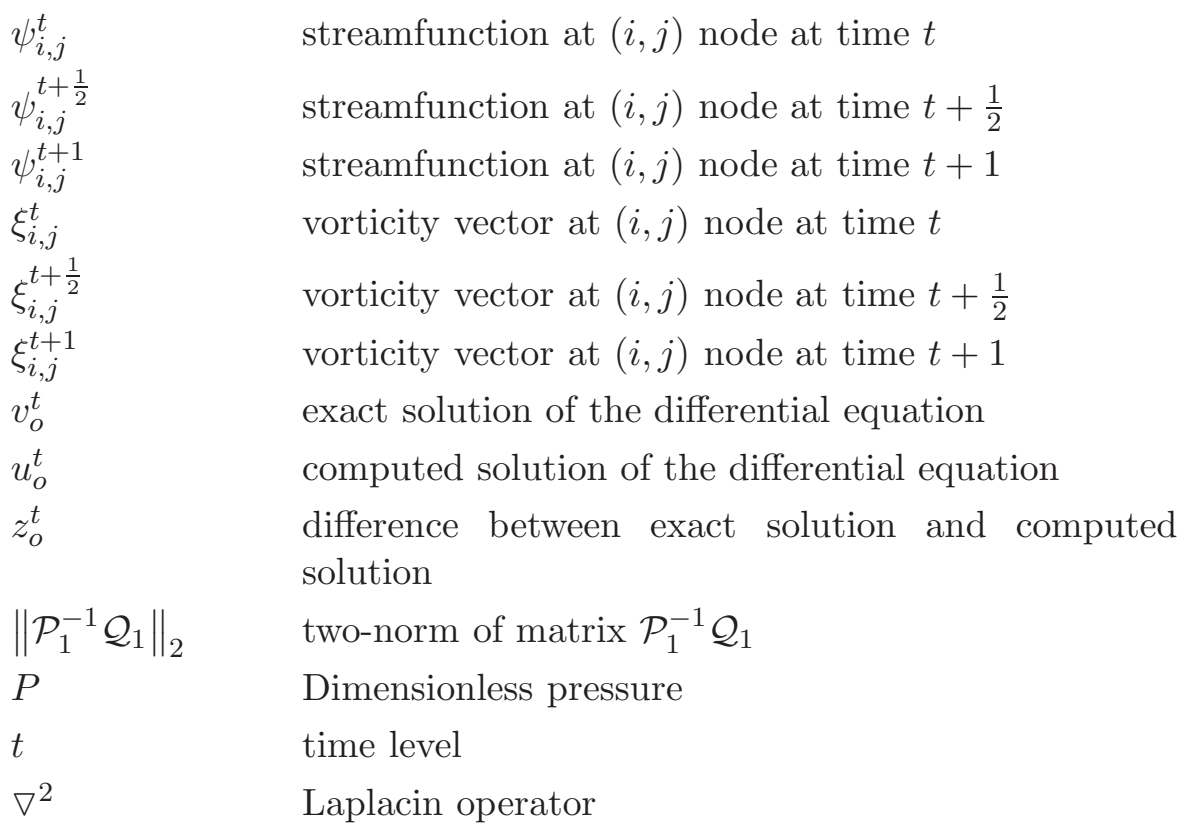

\section{Greek symbols}

$\begin{array}{ll}\lambda_{p s} & \text { eigenvalues of matrix } \mathcal{P}_{1} \\ \lambda_{q s} & \text { eigenvalues of matrix } \mathcal{Q}_{1} \\ \psi & \text { streamfunction } \\ \xi & \text { vorticityfunction } \\ \rho(A) & \text { spectral radius of } A, \text { which is the maximum of the } \\ & \text { moduli of its eigenvalues } \lambda_{i}, i=1,2, \ldots, n\end{array}$

\section{Acknowledgements}

The first and second authors acknowledge the financial support received from the University of Delhi under Research and Development Scheme 2015-16 for providing research grant vide letter no.RC/2015/9677 dated $15^{\text {th }}$ October 2015 as well as Non-Net Fellowship to M.Phil. Scholars vide letter No. Ref.NO.Schs/ Non-Net/ 139/EXT-145/2015-16/254, Dated: 11 ${ }^{\text {th }}$ April,2015.

\section{References}

[1] U. Ghia, K.N. Ghia and C.T. Shin, High-Re solutions for incompressible 
flow using the Navier-Stokes equations and a multigrid method, Journal of Computational Physics, 48 (1982), 387-411.

[2] R. Schreiber and H.B. Keller, Driven cavity flow by efficient numerical techniques, Journal of Computational Physics, 49 (1983), 310-333.

[3] G.D. Smith, Numerical Solution of Partial Differential Equations: Finite Difference Methods, Oxford University Press, New York, U.S.A. (1985).

[4] T. Fusegi and Bakhtierfarouk, Predictions of fluid flow and heat transfer problems by the vorticity-velocity formulation of the Navier-Stokes equations, Journal of Computational Physics, 65 (1986), 227-243.

[5] S.C. Chapra and R.P. Canale, Numerical Methods for Engineers, McGrawHill Co., New York (1989).

[6] C.H. Bruneau and C. Jouron, An efficient scheme for solving steady incompressible Navier-Stokes equations, Journal of Computational Physics, 89 (1990), 389-413.

[7] T.E. Tezduyar, J. Liou, D.K. Ganjoo and M. Behr, Solution techniques for the vorticity-streamfunction formulation of two-dimensional unsteady incompressible flows, International Journal for Numerical Methods in Fluids, 11 (1990), 515-539.

[8] C.K. Aidun, N.G. Triantafillopouios and J.D. Benson, Global stability of a lid-driven cavity with through flow: flow visualization studies, Phys. Fluids A, 3 (1991), 2081-2091.

[9] D.A. Zumbrunnen, K.C. Miles and Y.H. Liu, Auto-processing of very fine scale composite materials by chotic mixing of melts, Composites Part A, 27A (1995), 37-47.

[10] P.S. Ghoshdastidar, Computer Simulation of Flow and Heat Transfer, Tata McGraw-Hill Publishing Co. Ltd., New Delhi (1998).

[11] P.N. Shankar and M.D. Deshpande, Fluid mechanics in the driven cavity, Annual Review of Fluid Mechanics, 32 (2000), 93-136.

[12] J.C. Kalita, D.C. Dalal and A.K. Dass, Fully compact higher order computation of steady-state natural convection in a square cavity, Physical Review E, 64 (2001), 1-13. 
[13] J.C. Kalita, D.C. Dalal and A.K. Dass, A transformation-free HOC scheme for steady convection-diffusion on non-uniform grids, International Journal for Numerical Methods in Fluids, 44 (2004), 33-53.

[14] H. Shokouhmand and H. Sayehvand, Numerical study of flow and heat transfer in a square driven cavity, International Journal of Engineering Transactions A: Basics, 17 (2004), 301-317.

[15] E. Erturk, T.C. Corke and C. Gokcol, Numerical Solutions of 2-D steady incompressible driven cavity flow at high Reynolds numbers, International Journal for Numerical Methods in Fluids, 48 (2005), 747-774.

[16] C.H. Bruneau and M. Saad, The 2-D lid-driven cavity problem revisited, Computers and Fluids, 35 (2006), 326-348.

[17] A. Salem, On the numerical solution of the incompressible Navier-Stokes equations in primitive variables using grid generation techniques, Mathematical and Computational Applications, 11 (2006), 127-136.

[18] J.C. Kalita and S. Sen, The $(9,5)$ HOC formulation for the transient Navier-Stokes equations in primitive variable, International Journal for Numerical Methods in Fluids, 55 (2007), 387-406.

[19] J.M. McDonough, Lectures on Computational Numerical Analysis of Partial Differential Equations, Available et: http://www.engr.uky.edu/ acfd (2007).

[20] K. Muralidhar and T. Sundararajan, Computational Fluid Flow and Heat Transfer, 2nd ed., Narosa, New Delhi (2008).

[21] E. Erturk, Discussions on driven cavity flow, International Journal for Numerical Methods in Fluids, 60 (2009), 275-294.

[22] T.Y. Hou, Stable fourth-order stream-function methods for incompressible flows with boundaries, Journal of Computational Mathematics, 27 (2009), 441-458.

[23] S. Biringen and C. Y. Chow, An Introduction to Computational Fluid Mechanics by Examples, John Wiley and Sons, Inc., Hoboken, New Jersey (2011).

[24] K. Poochinapan, Numerical implementations for 2-d lid-driven cavity flow in stream function formulation, International Scholarly Research network 
ISRN Applied Mathematics, 2012 (2012), Article ID 871538, 17 pp., doi:10.5402/2012/871538.

[25] K.M. Salah Uddin and L.K. Saha, Numerical solution of 2-d incompressible driven cavity flow with wavy bottom surface, American Journal of Applied Mathematics, 3, No 1-1 (2015), 30-42.

[26] D.W. Peaceman and H.H. Rachford, The numerical solution of parabolic and elliptic differential equations, Journal of the Society for Industrial and Applied Mathematics, 3 (1955), 28-41. 
